TRANSACTIONS OF THE

AMERICAN MATHEMATICAL SOCIETY

Volume 356, Number 5, Pages 1937-1955

S 0002-9947(03)03353-1

Article electronically published on June 24, 2003

\title{
SIMPLE BRATTELI DIAGRAMS WITH A GÖDEL-INCOMPLETE C*-EQUIVALENCE PROBLEM
}

\author{
DANIELE MUNDICI
}

\begin{abstract}
An abstract simplicial complex is a finite family of subsets of a finite set, closed under subsets. Every abstract simplicial complex $\mathcal{C}$ naturally determines a Bratteli diagram and a stable AF-algebra $A(\mathcal{C})$. Consider the following problem:

INPUT: a pair of abstract simplicial complexes $\mathcal{C}$ and $\mathcal{C}^{\prime}$;

QUESTION: is $A(\mathcal{C})$ isomorphic to $A\left(\mathcal{C}^{\prime}\right)$ ?

We show that this problem is Gödel incomplete, i.e., it is recursively enumerable but not decidable. This result is in sharp contrast with the recent decidability result by Bratteli, Jorgensen, Kim and Roush, for the isomorphism problem of stable AF-algebras arising from the iteration of the same positive integer matrix. For the proof we use a combinatorial variant of the De Concini-Procesi theorem for toric varieties, together with the Baker-Beynon duality theory for lattice-ordered abelian groups, Markov's undecidability result, and Elliott's classification theory for AF-algebras.
\end{abstract}

\section{INTRODUCTION}

Every abstract simplicial complex $\mathcal{C}$ over $\{1,2, \ldots, n\}$ naturally generates a direct system

$$
D(\mathcal{C})=\left\{\varphi_{i}: \mathbf{Z}^{n+i} \rightarrow \mathbf{Z}^{n+i+1} \mid i=0,1, \ldots\right\}
$$

of simplicial groups and positive homomorphisms, by repeated application of the De Concini-Procesi starring procedure [18], 8]. Elliott's classification [6], 11] yields from $D(\mathcal{C})$ a stable AF-algebra $A(\mathcal{C})$, and we say that $\mathcal{C}$ is $C^{*}$-equivalent to $\mathcal{C}^{\prime}$ iff $A(\mathcal{C})$ is isomorphic to $A\left(\mathcal{C}^{\prime}\right)$. We prove the Gödel-incompleteness (i.e., the undecidability together with the effective enumerability) of the isomorphism problem for stable AF-algebras arising from abstract finite simplicial complexes. While the isomorphism problem is decidable for stable AF-algebras arising from the iteration of the same positive integer matrix [4], [5], our undecidability result holds for a class $L$ of stable AF-algebras arising from very simple sequences of matrices $\varphi_{i}$ obtained by appending a suitable bottom row of zeros and ones to the $(n+i) \times(n+i)$ identity matrix. A stable AF-algebra is in $L$ iff its $K_{0}$-group, equipped with the natural order induced by the Murray-von Neumann order of equivalence classes of

Received by the editors March 12, 2002 and, in revised form, March 31, 2003.

2000 Mathematics Subject Classification. Primary 46L35, 06F20, 20F10, 03D40, 52B20.

Key words and phrases. Isomorphism of Bratteli diagrams, stable AF-algebra, Elliott's classification, Markov undecidability theorem, $C^{*}$-equivalence, De Concini-Procesi theorem for toric varieties.

Partially supported by MURST Project on Logic. 
projections, is a finitely generated projective lattice-ordered abelian group. Undecidability follows from Markov's celebrated theorem on the undecidability of the piecewise linear homeomorphism of compact simplicial complexes, [9].

\section{Statement of the Main Result}

For each $n=1,2, \ldots$, elements of $\operatorname{Hom}\left(\mathbf{Z}^{n}, \mathbf{Z}\right)$ are identified with row (= bra) vectors $\mathbf{h}=\left(h_{1}, \ldots, h_{1}\right) \in \mathbf{Z}^{n}$. For every column (= ket) vector $\mathbf{x} \in \mathbf{Z}^{n}$, the value $\mathbf{h}(\mathbf{x})$ is given by the scalar product $\mathbf{h} \circ \mathbf{x}$. Given ket vectors $\mathbf{x} \neq \mathbf{y} \in \mathbf{Z}^{n}$, the $\mathbf{h}$-temperature of $\{\mathbf{x}, \mathbf{y}\}$ is the integer $(\mathbf{h} \circ \mathbf{x})(\mathbf{h} \circ \mathbf{y})$. We say that $\{\mathbf{x}, \mathbf{y}\}$ is $\mathbf{h}$-cooler than $\left\{\mathbf{x}^{\prime}, \mathbf{y}^{\prime}\right\}$ iff $(\mathbf{h} \circ \mathbf{x})(\mathbf{h} \circ \mathbf{y})<\left(\mathbf{h} \circ \mathbf{x}^{\prime}\right)\left(\mathbf{h} \circ \mathbf{y}^{\prime}\right)$.

Throughout this paper, the set $\operatorname{Hom}\left(\mathbf{Z}^{n}, \mathbf{Z}\right)$ is equipped with the following lexicographic order: Given distinct $\mathbf{h}, \mathbf{k} \in \operatorname{Hom}\left(\mathbf{Z}^{n}, \mathbf{Z}\right)$, we say that $\mathbf{h}$ precedes $\mathbf{k}$, in symbols $\mathbf{h} \sqsubset \mathbf{k}$, iff either $\left|h_{1}\right|+\cdots+\left|h_{n}\right|<\left|k_{1}\right|+\cdots+\left|k_{n}\right|$, or $\left|h_{1}\right|+\cdots+\left|h_{n}\right|=$ $\left|k_{1}\right|+\cdots+\left|k_{n}\right|$ and, letting $i$ be the first index such that $h_{i} \neq k_{i}$, we have $h_{i}<k_{i}$. One similarly defines the lexicographic order for two-element subsets of $\mathbf{Z}^{n}$.

Let $\Delta$ be a (finite) ${ }^{1}$ non-singular fan in $\mathbf{R}^{n}$ (i.e., 18, $\Delta$ is a complex of rational simplicial cones in $\mathbf{R}^{n}$ such that every cone is generated by a part of a basis of $\mathbf{Z}^{n}$ ). Let $\Delta^{(i)}$ denote the set of $i$-dimensional cones in $\Delta$. Let us write

$$
\Delta^{(1)}=\left\{\rho_{1}, \ldots, \rho_{k}\right\}=\left\{\mathbf{R}_{\geq 0} \mathbf{v}_{1}, \ldots, \mathbf{R}_{\geq 0} \mathbf{v}_{k}\right\}=\left\{\left\langle\mathbf{v}_{1}\right\rangle, \ldots,\left\langle\mathbf{v}_{k}\right\rangle\right\},
$$

and say that each $\rho_{i}$ is generated by the primitiv ${ }^{2}$ vector $\mathbf{v}_{i} \in \mathbf{Z}^{n}$. The latter is uniquely determined. We shall similarly express every two-dimensional cone $\sigma=\left\langle\mathbf{v}_{i}, \mathbf{v}_{j}\right\rangle \in \Delta^{(2)}$ as the positive real span of its (uniquely determined) primitive generators $\mathbf{v}_{i}$ and $\mathbf{v}_{j} \in \mathbf{Z}^{n}$.

Suppose $\emptyset \neq \Delta^{(2)}$. Let $\mathbf{h} \in \mathbf{Z}^{n}$ be the $\sqsubset$-first bra vector such that for some two-dimensional cone $\sigma=\left\langle\mathbf{v}_{i}, \mathbf{v}_{j}\right\rangle \in \Delta^{(2)}$, the $\mathbf{h}$-temperature of $\left\{\mathbf{v}_{i}, \mathbf{v}_{j}\right\}$ is $<0$. Let $\left\{\mathbf{v}_{i^{*}}, \mathbf{v}_{j^{*}}\right\}$ have the smallest $\mathbf{h}$-temperature. Then the successor $\Delta^{\uplus}$ of $\Delta$ is the star subdivision of $\Delta$ obtained by starring $\Delta$ at the mediant $\mathbf{v}_{k+1}=\mathbf{v}_{i^{*}}+\mathbf{v}_{j^{*}}$ of it s $^{3}$ $\mathbf{h}$-coolest cone $\sigma^{*}=\left\langle\mathbf{v}_{i^{*}}, \mathbf{v}_{j^{*}}\right\rangle \in \Delta^{(2)}$. In other words, $\Delta^{\uplus}$ has a new 1-dimensional cone $\left\langle\mathbf{v}_{k+1}\right\rangle$, and every cone $\sigma=\left\langle\mathbf{v}_{i^{*}}, \mathbf{v}_{j^{*}}, \ldots\right\rangle$ in $\Delta$ containing $\sigma^{*}$ as a face is replaced in $\Delta^{\uplus}$ by the two cones $\sigma^{\prime}=\left\langle\mathbf{v}_{k+1}, \mathbf{v}_{j^{*}}, \ldots\right\rangle$ and $\sigma^{\prime \prime}=\left\langle\mathbf{v}_{k+1}, \mathbf{v}_{i^{*}}, \ldots\right\rangle$. Note that $\Delta^{\uplus}$ is a non-singular fan in $\mathbf{R}^{n}$.

The transition matrix $\varphi$ from $\Delta$ to its successor $\Delta^{\uplus}$ is obtained by appending to the $k \times k$ identity matrix $\left(\delta_{i j}\right)$ a bottom row whose entries are 0 , with the exception of the $i^{*}$ th and $j^{*}$ th entries, which are equal to 1 .

For each integer $n \geq 2$, an abstract simplicial complex $\mathcal{C}$ over $\{1, \ldots, n\}$ is a collection of subsets of $\{1, \ldots, n\}$ whose union is $\{1, \ldots, n\}$, and such that, whenever $X \in \mathcal{C}$ and $\emptyset \neq Y \subseteq X$, then $Y \in \mathcal{C}$. We say that $\mathcal{C}$ is non-degenerate iff it has at least one set with two elements 4

Let $\mathcal{C}$ be a non-degenerate abstract simplicial complex over $\{1,2, \ldots, n\}$. Let $\mathbf{e}_{1}=(1,0,0, \ldots, 0), \ldots, \mathbf{e}_{n}=(0,0,0, \ldots, 1)$ be the standard basis vectors in $\mathbf{Z}^{n}$. The map sending each $i=1,2, \ldots, n$ into $\mathbf{e}_{i}$ transforms $\mathcal{C}$ into a non-singular fan

\footnotetext{
${ }^{1}$ In this paper, complexes and fans are always assumed to be finite.

${ }^{2}$ In the sense that the greatest common divisor of the coordinates of $\mathbf{v}_{i}$ is equal to 1 .

${ }^{3}$ If several sets $\left\{\mathbf{v}_{i}, \mathbf{v}_{j}\right\}$ have the same minimal $\mathbf{h}$-temperature, we choose the first one, according to the above lexicographic order of two-element subsets of $\mathbf{Z}^{n}$.

${ }^{4}$ Every abstract simplicial complex considered in this paper will be tacitly defined over some finite initial segment of the set $\{1,2,3, \ldots\}$.
} 
$\Delta(\mathcal{C})$ in $\mathbf{R}^{n}$, as follows: each one-dimensional cone of $\Delta(\mathcal{C})$ has the form $\rho_{i}=\left\langle\mathbf{e}_{i}\right\rangle$; each 2-dimensional cone of $\Delta(\mathcal{C})$ has the form $\left\langle\mathbf{e}_{i}, \mathbf{e}_{j}\right\rangle$ whenever the set $\{i, j\}$ belongs to $\mathcal{C}$, and so on. $\Delta(\mathcal{C})$ is called the initial non-singular fan of $\mathcal{C}$. The diagram $D(\mathcal{C})$ is the sequence of $(0,1)$ matrices $\varphi_{0}, \varphi_{1}, \varphi_{2}, \ldots$, where $\varphi_{i}$ is the transition matrix from $\Delta_{i}$ to its successor $\Delta_{i+1}=\Delta_{i}^{\uplus}\left(\right.$ and $\left.\Delta_{0}=\Delta(\mathcal{C})\right)$. $D(\mathcal{C})$ is a short notation for the direct system

$$
\mathbf{Z}^{n} \stackrel{\varphi_{0}}{\longrightarrow} \mathbf{Z}^{n+1} \stackrel{\varphi_{1}}{\longrightarrow} \mathbf{Z}^{n+2} \stackrel{\varphi_{2}}{\longrightarrow} \cdots
$$

of simplicia 5 groups and order-preserving homomorphisms. We denote by $\lim D(\mathcal{C})$ the direct limit of the above system, in the category of partially ordered $\vec{a}$ belian groups with order-preserving homomorphisms [6], [11. Two non-degenerate abstract simplicial complexes $\mathcal{C}$ over $\{1,2, \ldots, n\}$, and $\mathcal{C}^{\prime}$ over $\left\{1,2, \ldots, n^{\prime}\right\}$ are said to be $C^{*}$-equivalent iff $\lim _{\rightarrow} D(\mathcal{C}) \cong \lim _{\rightarrow} D\left(\mathcal{C}^{\prime}\right)$ 6

The main aim of this paper is to prove the following:

Theorem 1.1. (i) There is no Turing machine which, having as input two nondegenerate abstract simplicial complexes $\mathcal{C}$ over $\{1,2, \ldots, n\}$ and $\mathcal{C}^{\prime}$ over $\{1,2, \ldots$, $\left.n^{\prime}\right\}$, decides whether they are $C^{*}$-equivalent.

(ii) There is a Turing machine which enumerates precisely all pairs $\left(\mathcal{C}, \mathcal{C}^{\prime}\right)$ of $C^{*}$-equivalent non-degenerate abstract simplicial complexes.

\section{RADiant FUnCtions AND Schauder hats}

A homogeneous linear polynomial with integer coefficients is a function $l: \mathbf{R}^{n} \rightarrow$ $\mathbf{R}$ of the form $l\left(x_{1}, \ldots, x_{n}\right)=a_{1} x_{1}+\cdots+a_{n} x_{n}$, where the $a_{j}$ 's are integers.

A function $f: \mathbf{R}^{n} \rightarrow \mathbf{R}$ is said to be radian 7 iff it is continuous and there is a finite set $\left\{l_{1}, \ldots, l_{q}\right\}$ of homogeneous linear polynomials with integer coefficients such that, for every $\mathbf{x}=\left(x_{1}, \ldots, x_{n}\right) \in \mathbf{R}^{n}$, there is an index $i \in\{1, \ldots, q\}$ satisfying $f(\mathbf{x})=l_{i}(\mathbf{x})$.

For any subset $X \subseteq \mathbf{R}^{n}$ we say that a function $g: X \rightarrow \mathbf{R}$ is radiant over $X$ iff $g$ is the restriction to $X$ of a radiant function $f$ over $\mathbf{R}^{n}$. We let $\operatorname{Radiant}(X)$ denote the group of radiant functions over $X$ with the natural pointwise operations and order 8

For every complex $\Gamma$ of cones in $\mathbf{R}^{n}$, the support $|\Gamma| \subseteq \mathbf{R}^{n}$ of $\Gamma$ is the point-set union of all cones in $\Gamma$, in symbols, $|\Gamma|=\bigcup\{\sigma \mid \sigma \in \Gamma\}$.

Following [18, p. 66], for any non-singular fan $\Delta$ in $\mathbf{R}^{n}$ a function $s:|\Delta| \rightarrow \mathbf{R}$ is said to be a $\Delta$-linear support function iff $s$ is integer-valued over $|\Delta| \cap \mathbf{Z}^{n}$ and is linear over each cone $\sigma \in \Delta$. We denote by $\operatorname{SF}\left(\mathbf{Z}^{n}, \Delta\right)$ the additive group of all $\Delta$-linear support functions (with natural pointwise order).

Proposition 2.1. Let $\Delta$ be a non-singular fan in $\mathbf{R}^{n}$, and let

$$
\left\{\rho_{1}, \ldots, \rho_{k}\right\}=\left\{\left\langle\mathbf{v}_{1}\right\rangle, \ldots,\left\langle\mathbf{v}_{k}\right\rangle\right\}
$$

\footnotetext{
${ }^{5}$ By definition, the simplicial order of $\mathbf{Z}^{n}$ is the (coordinatewise) product order induced by the natural order of $\mathbf{Z}$.

${ }^{6}$ This terminology will be justified in the final section of this paper.

${ }^{7}$ Also known in the literature as continuous piecewise linear homogeneous with integer coefficients.

${ }^{8}$ In [9] the same set of functions is denoted $\mathrm{PI}(X)$.
} 
be the list of 1-dimensional cones of $\Delta$. Let the map $\eta: S F\left(\mathbf{Z}^{n}, \Delta\right) \rightarrow \mathbf{Z}^{k}$ be defined by

$$
\eta(s)=\left(s\left(\mathbf{v}_{1}\right), \ldots, s\left(\mathbf{v}_{k}\right)\right),
$$

for each function $s \in \operatorname{SF}\left(\mathbf{Z}^{n}, \Delta\right)$. Then $\eta$ is an isomorphism of $\operatorname{SF}\left(\mathbf{Z}^{n}, \Delta\right)$ onto the abelian group $\mathbf{Z}^{k}$,

$$
\eta: \mathrm{SF}\left(\mathbf{Z}^{n}, \Delta\right) \cong \mathbf{Z}^{k}
$$

Once the latter group is equipped with the coordinatewise (simplicial) order given by the natural order of $\mathbf{Z}$, it follows that $\eta$ preserves the natural pointwise order of functions in $\mathrm{SF}\left(\mathbf{Z}^{n}, \Delta\right)$, and $\eta^{-1}$ preserves the order of $\mathbf{Z}^{k}$; in symbols,

$$
\eta:\left(\operatorname{SF}\left(\mathbf{Z}^{n}, \Delta\right)\right)^{+} \cong\left(\mathbf{Z}^{+}\right)^{k} .
$$

Proof. Routine. (Compare with [18, p. 67].)

Proposition 2.2. Let $\Delta$ be a non-singular fan in $\mathbf{R}^{n}$ and $\rho=\langle\mathbf{v}\rangle$ a 1-dimensional cone of $\Delta$ with primitive generating vector $\mathbf{v} \in \mathbf{Z}^{n}$. Then there exists precisely one $\Delta$-linear support function $h_{\rho}$ over $|\Delta|$ which is determined by the following stipulations:

(a) $h_{\rho}(\mathbf{v})=1$;

(b) $h_{\rho}(\mathbf{w})=0$, if $\mathbf{w}$ is the primitive generating vector of a 1-dimensional cone of $\Delta$ other than $\rho$.

Proof. Immediate from Proposition 2.1.

Each function $h_{\rho}$ is said to be the Schauder hat of $\rho$ in $\Delta$. We denote by $\mathcal{H}(\Delta)$ the set of Schauder hats of all 1-dimensional cones in $\Delta$. Our present Schauder hats are a homogeneous reformulation of the functions defined in [15] in an MV-algebraic context.

Proposition 2.3. Let $\Delta$ be a non-singular fan in $\mathbf{R}^{n}$, and let $\mathbf{v}_{1}, \ldots, \mathbf{v}_{k}$ be the primitive generating vectors of the 1-dimensional cones of $\Delta$, with their corresponding Schauder hats $h_{1}=h_{\left\langle\mathbf{v}_{1}\right\rangle}, \ldots, h_{k}=h_{\left\langle\mathbf{v}_{k}\right\rangle}$. Then:

(i) For each $j=1, \ldots, k, h_{j}$ is a radiant function over $|\Delta|$.

(ii) The isomorphism $\eta$ of Proposition [2.1 maps $\mathcal{H}(\Delta)$ one-to-one onto the atoms (i.e., the minimal nonzero elements) of the simplicial group $\mathbf{Z}^{k}$.

(iii) The Schauder hats $h_{1}, \ldots, h_{k}$ are linearly independent in $\operatorname{SF}\left(\mathbf{Z}^{n}, \Delta\right)$. Every function $s \in \operatorname{SF}\left(\mathbf{Z}^{n}, \Delta\right)$ is uniquely expressible as a linear combination of the Schauder hats of $\mathcal{H}(\Delta)$ with integer coefficients.

Proof. (i) As shown, e.g., in [8 Theorem 9.3(b), p. 257], $\Delta$ can be extended to a non-singular fan $\Delta^{\diamond}$ with support $\mathbf{R}^{n}$. In symbols, $\Delta \subseteq \Delta^{\diamond}$ and $\left|\Delta^{\diamond}\right|=\mathbf{R}^{n}$. Let $\mathcal{H}\left(\Delta^{\diamond}\right)$ be the family of Schauder hats of all 1-dimensional cones of $\Delta^{\diamond}$. For every Schauder hat $h \in \mathcal{H}\left(\Delta^{\diamond}\right)$, the restriction of $h$ to $|\Delta|$ is either the zero function over $|\Delta|$, or else it is a Schauder hat in $\mathcal{H}(\Delta)$. In this way we obtain all Schauder hats in $\mathcal{H}(\Delta)$.

(ii) By definition, for each $j=1, \ldots, k$,

$$
\eta\left(h_{j}\right)=\left(h_{j}\left(\mathbf{v}_{1}\right), \ldots, h_{j}\left(\mathbf{v}_{k}\right)\right)=\left(\delta_{j 1}, \ldots, \delta_{j k}\right)=\mathbf{e}_{j} .
$$

Now, the standard basis vectors of $\mathbf{Z}^{k}$ are precisely the atoms of the simplicial group $\mathbf{Z}^{k}$.

(iii) Note that only $h_{j}$ is nonzero at $\mathbf{v}_{j}$, and then use (i) and (ii). 
Proposition 2.4. Let $\Delta$ and $\Delta^{*}$ be non-singular fans in $\mathbf{R}^{n}$, with $\Delta^{*}$ being a subdivision of $\Delta$. In other words, $\left|\Delta^{*}\right|=|\Delta|$ and every cone of $\Delta$ is the union of cones of $\Delta^{*}$. Let $\mathbf{v}_{1}, \ldots, \mathbf{v}_{k}$ be the primitive generating vectors of all 1-dimensional cones of $\Delta$, with their corresponding Schauder hats $h_{1}=h_{\left\langle\mathbf{v}_{1}\right\rangle}, \ldots, h_{k}=h_{\left\langle\mathbf{v}_{k}\right\rangle}$. Similarly let $\mathbf{w}_{1}, \ldots, \mathbf{w}_{k^{*}}$ be the primitive generating vectors of all 1-dimensional cones of $\Delta^{*}$, with Schauder hats $h_{1}^{*}, \ldots, h_{k^{*}}^{*}$. For each $1 \leq j \leq k$ and $1 \leq i \leq k^{*}$, let $m_{i j}=h_{j}\left(\mathbf{w}_{i}\right)$. Then:

(i) Each $m_{i j}$ is an integer $\geq 0$.

(ii) Each Schauder hat $h_{j}$ is uniquely expressible as a linear combination of the $h_{i}^{*}$ 's with positive integer coefficients, as follows:

$$
h_{j}=\sum_{1 \leq i \leq k^{*}} m_{i j} h_{i}^{*} \quad(1 \leq j \leq k) .
$$

(iii) In particular, when $\Delta^{*}$ coincides with the successor $\Delta^{\uplus}$ of $\Delta$, and is obtained, say, by starring $\Delta$ at the mediant $\mathbf{v}_{p}+\mathbf{v}_{q}$ of its 2-dimensional cone $\sigma=\left\langle\mathbf{v}_{p}, \mathbf{v}_{q}\right\rangle$, then the matrix $\left(m_{i j}\right)$ is obtained by appending to the $k \times k$ identity matrix a bottom row with zero entries, except the pth and the qth, which are equal to 1.

Proof. (i) Immediate by definition.

(ii) For each $1 \leq j \leq k$ both functions $h_{j}$ and $\sum_{1 \leq i \leq k^{*}} m_{i j} h_{i}^{*}$ are homogeneous linear over each cone of $\Delta^{*}$. Since both functions are continuous, in order to prove that they coincide over all of $|\Delta|=\left|\Delta^{*}\right|$, it is enough to check that they coincide at the primitive generating vector $\mathbf{w}_{i}$ of every 1-dimensional cone of $\Delta^{*}$, where $i=1, \ldots, k^{*}$. This is immediate by construction. Uniqueness follows from Proposition 2.3(iii).

(iii) By definition of star subdivision, $k^{*}=k+1, \mathbf{v}_{1}=\mathbf{w}_{1}, \ldots, \mathbf{v}_{k}=\mathbf{w}_{k}$, and $\Delta^{*}$ has the same 1-dimensional cones as $\Delta$, plus the new 1-dimensional cone $\left\langle\mathbf{w}_{k^{*}}\right\rangle=\left\langle\mathbf{v}_{p}+\mathbf{v}_{q}\right\rangle$. In case $i<k^{*}$ we can write $m_{i j}=h_{j}\left(\mathbf{w}_{i}\right)=h_{j}\left(\mathbf{v}_{i}\right)=\delta_{i j}$. In case $i=k^{*}$ we have $m_{k^{*} j}=h_{j}\left(\mathbf{w}_{k^{*}}\right)=h_{j}\left(\mathbf{v}_{p}+\mathbf{v}_{q}\right)=h_{j}\left(\mathbf{v}_{p}\right)+h_{j}\left(\mathbf{v}_{q}\right)=\delta_{j p}+\delta_{j q}$, as required.

\section{From abstract COMPLEXES to Diagrams}

An abelian group $G$ is said to be partially ordered iff it is equipped with a subset $P$ such that $P+P=P, P-P=G$, and $P \cap-P=\emptyset$. We write $x \leq y$ iff $y-x \in P$. We say that $(G, P)$ is unperforated iff, whenever $a \in G$ and $n a \in P$ for some integer $n>0$, then $a \in P$. We call $P$ the positive cone of $G$, and write $G^{+}$instead of $P$. A morphism in the category of partially ordered abelian groups is an order-preserving group homomorphism.

By a dimension group $(6],[11])$ we mean a partially ordered unperforated abelian group with the Riesz interpolation property: given $a_{1}, a_{2}, b_{1}, b_{2} \in G$ with $a_{i} \leq$ $b_{j}(i, j \in\{1,2\})$, there is $c \in G$ with $a_{i} \leq c \leq b_{j}(i, j \in\{1,2\})$.

In this section we shall prove

Theorem 3.1. Let $\mathcal{C}$ be a non-degenerate abstract simplicial complex over $\{1,2$, $\ldots, n\}, \Delta(\mathcal{C})=\Delta_{0}$ its initial non-singular fan, $\Delta_{0}, \Delta_{1}, \Delta_{2}, \ldots$ the sequence of successor fans, $\varphi_{i}$ the transition matrix from $\Delta_{i}$ to $\Delta_{i+1}=\Delta_{i}^{\uplus}$, and

$$
D(\mathcal{C})=\left\{\mathbf{Z}^{n+i} \stackrel{\varphi_{i}}{\longrightarrow} \mathbf{Z}^{n+i+1} \mid i=0,1, \ldots\right\}
$$


the diagram of $\mathcal{C}$. Then, in the category of partially ordered abelian groups, we have the isomorphism

$$
\lim _{\rightarrow} D(\mathcal{C}) \cong \operatorname{Radiant}(|\Delta(\mathcal{C})|)
$$

For the proof we need

Lemma 3.2. For each $i=0,1, \ldots$ we have a commutative diagram

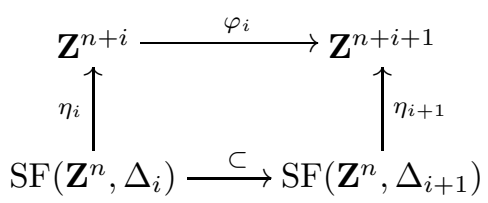

in the sense that, for all $f \in \operatorname{SF}\left(\mathbf{Z}^{n}, \Delta_{i}\right), \varphi_{i}\left(\eta_{i}(f)\right)=\eta_{i+1}(f)$.

Proof. In the light of Propositions 2.12.4 we can safely restrict attention to the Schauder hats $h_{\left\langle\mathbf{v}_{1}\right\rangle}, \ldots, h_{\left\langle\mathbf{v}_{k}\right\rangle}$ of $\Delta_{i}$. Assume $\Delta_{i+1}$ is obtained by starring $\Delta_{i}$ at the mediant $\mathbf{w}_{k+1}=\mathbf{v}_{p}+\mathbf{v}_{q}$ of the 2-dimensional cone $\left\langle\mathbf{v}_{p}, \mathbf{v}_{q}\right\rangle$ of $\Delta_{i}$. Let $h_{\left\langle\mathbf{w}_{1}\right\rangle}^{\prime}, \ldots, h_{\left\langle\mathbf{w}_{k}\right\rangle}^{\prime}, h_{\left\langle\mathbf{w}_{k+1}\right\rangle}^{\prime}$ be the Schauder hats of the successor fan $\Delta_{i}^{\uplus}=\Delta_{i+1}$, where $\mathbf{w}_{1}=\mathbf{v}_{1}, \ldots, \mathbf{w}_{k}=\mathbf{v}_{k}$ and $\mathbf{w}_{k+1}=\mathbf{v}_{p}+\mathbf{v}_{q}$. Then for each $j=1, \ldots, k$ we have the following maps:

$$
h_{\left\langle\mathbf{v}_{j}\right\rangle} \stackrel{\eta_{i}}{\rightarrow} \mathbf{e}_{j} \stackrel{\varphi_{i}}{\longrightarrow} j \text { th column of } \varphi_{i} \stackrel{\eta_{i+1}^{-1}}{\longrightarrow} h_{\left\langle\mathbf{w}_{j}\right\rangle}^{\prime}+\delta_{j p} h_{\left\langle\mathbf{w}_{k+1}\right\rangle}^{\prime}+\delta_{j q} h_{\left\langle\mathbf{w}_{k+1}\right\rangle}^{\prime}=h_{\left\langle\mathbf{v}_{j}\right\rangle} .
$$

Lemma 3.3. For each $j=0,1, \ldots$, the homomorphism $\varphi_{j}: \mathbf{Z}^{n+j} \rightarrow \mathbf{Z}^{n+j+1}$ has the following properties:

(i) $\varphi_{j}$ is injective.

(ii) The image $\varphi_{j}\left(\mathbf{Z}^{n+j}\right) \subseteq \mathbf{Z}^{n+j+1}$ is a pure subgroup of $\mathbf{Z}^{n+j+1}$.

(iii) $\varphi_{j}$ is order-preserving, in the sense that $\varphi_{j}\left(\left(\mathbf{Z}^{+}\right)^{n+j}\right) \subseteq\left(\mathbf{Z}^{+}\right)^{n+j+1}$.

(iv) As an order-preserving map, $\varphi_{j}$ is strong, in the sense that whenever $\mathbf{x} \notin$ $\left(\mathbf{Z}^{+}\right)^{n+j}$, then $\varphi_{j}(\mathbf{x}) \notin\left(\mathbf{Z}^{+}\right)^{n+j+1}$.

Proof. By direct inspection.

Lemma 3.4. For each $i=0,1,2, \ldots$ the group of $\Delta_{i}$-linear support functions $\operatorname{SF}\left(\mathbf{Z}^{n}, \Delta_{i}\right)$ is a subgroup of Radiant $(|\Delta(\mathcal{C})|)$. Furthermore,

$$
\left(\mathrm{SF}\left(\mathbf{Z}^{n}, \Delta_{i}\right)\right)^{+} \subseteq(\operatorname{Radiant}(|\Delta(\mathcal{C})|))^{+} .
$$

Proof. From Propositions 2.1 and 2.4.

Given any (bra) vector $\mathbf{h} \in \mathbf{Z}^{n}$, and any 2-dimensional $\sigma=\langle\mathbf{v}, \mathbf{w}\rangle \in \Delta_{i}$, we naturally define the $\mathbf{h}$-temperature of the cone $\sigma$ to be the integer $(\mathbf{h} \circ \mathbf{v})(\mathbf{h} \circ \mathbf{w})$.

Lemma 3.5. Let $\mathbf{h} \in \mathbf{Z}^{n}$ be a vector, and $i \geq 0$. If every cone $\sigma \in \Delta_{i}^{(2)}$ has $\mathbf{h}$-temperature $\geq 0$, then so does every cone $\tau \in \Delta_{i+1}^{(2)}$.

Proof. Immediate by definition of starring.

Lemma 3.6. For every vector $\mathbf{h} \in \mathbf{Z}^{n}$ there is an integer $d \geq 0$ such that every cone $\sigma=\langle\mathbf{v}, \mathbf{w}\rangle \in \Delta_{d}^{(2)}$ has $\mathbf{h}$-temperature $\geq 0$. 
Proof. Otherwise (absurdum hypothesis), let $\mathbf{h}$ be the $\sqsubset$-first counterexample. The existence of $\mathbf{h}$ is an immediate consequence of the definition of $\sqsubset$ given in Section 1 By Lemma 3.5, our absurdum hypothesis yields an integer $b \geq 0$ such that for all vectors $\mathbf{k} \sqsubset \mathbf{h}$ the $\mathbf{k}$-temperature of all cones of $\Delta_{b}^{(2)}$ is $\geq 0$, but there is no $d$ such that the $\mathbf{h}$-temperature of all cones of $\Delta_{d}^{(2)}$ is $\geq 0$.

Arguing as in the De Concini-Procesi theorem on elimination of points of indeterminacy [18, p. 39, and references therein], for each $i=b, b+1, b+2, \ldots$, we label $\Delta_{i}$ with the following pair of integers $(t(i), q(i))$ :

- $t(i)=\mathbf{h}$-temperature of the $\mathbf{h}$-coolest 2-dimensional cone of $\Delta_{i}$,

- $q(i)=$ number of 2 -dimensional cones of $\Delta_{i}$ having $\mathbf{h}$-temperature $t(i)$.

Since $\mathbf{h}$ is the $\sqsubset$-first bra vector such that for some 2-dimensional cone $\sigma \in \Delta_{i}$ the $\mathbf{h}$-temperature of $\sigma$ is $<0$, it follows that, by starring $\Delta_{i}$ at the mediant $\mathbf{m}=\mathbf{v}+\mathbf{w}$ of the h-coolest 2-dimensional cone $\sigma=\langle\mathbf{v}, \mathbf{w}\rangle \in \Delta_{i}$, we obtain the successor fan $\Delta_{i}^{\uplus}=\Delta_{i+1}$.

Claim: The non-singular fan $\Delta_{i+1}$ satisfies one of the following conditions:

- either $t(i+1)>t(i)$,

- or $t(i+1)=t(i)$ and $q(i+1)<q(i)$.

Indeed, by hypothesis, $(\mathbf{h} \circ \mathbf{v})(\mathbf{h} \circ \mathbf{w})=t(i)<0$. As explained in Section 1 any new cone $\tau$ in the successor fan $\Delta_{i+1}$ has either the form $\left\langle\mathbf{m}, \mathbf{w}, \mathbf{u}_{1}, \ldots, \mathbf{u}_{k}\right\rangle$ or the form $\left\langle\mathbf{m}, \mathbf{v}, \mathbf{u}_{1}, \ldots, \mathbf{u}_{k}\right\rangle$. To see that that the $\mathbf{h}$-temperature of $\tau$ is $>t(i)$, we first evaluate

$(\mathbf{h} \circ \mathbf{v})(\mathbf{h} \circ \mathbf{m})=(\mathbf{h} \circ \mathbf{v})(\mathbf{h} \circ \mathbf{v}+\mathbf{h} \circ \mathbf{w})=(\mathbf{h} \circ \mathbf{v})^{2}+(\mathbf{h} \circ \mathbf{v})(\mathbf{h} \circ \mathbf{w})>(\mathbf{h} \circ \mathbf{v})(\mathbf{h} \circ \mathbf{w})=t(i)$. Similarly, $(\mathbf{h} \circ \mathbf{w})(\mathbf{h} \circ \mathbf{m})>t(i)$. Further, for every $r=1, \ldots, k$, we can write

$$
\left(\mathbf{h} \circ \mathbf{u}_{r}\right)(\mathbf{h} \circ \mathbf{m})=\left(\mathbf{h} \circ \mathbf{u}_{r}\right)(\mathbf{h} \circ \mathbf{v}+\mathbf{h} \circ \mathbf{w})>t(i),
$$

because the two $\mathbf{h}$-temperatures $\left(\mathbf{h} \circ \mathbf{u}_{r}\right)(\mathbf{h} \circ \mathbf{v})$ and $\left(\mathbf{h} \circ \mathbf{u}_{r}\right)(\mathbf{h} \circ \mathbf{w})$ have opposite signs, unless $\left(\mathbf{h} \circ \mathbf{u}_{r}\right)=0$ (in which case, trivially, $\left.\left(\mathbf{h} \circ \mathbf{u}_{r}\right)(\mathbf{h} \circ \mathbf{m})>t(i)\right)$. The claim is settled.

Thus after a finite number $c$ of starrings we must reach a situation where the h-temperature of all 2-dimensional cones of $\Delta_{b+c}$ is $\geq 0$. We have obtained a contradiction, as required to complete the proof.

Lemma 3.7. For every function $0 \leq g \in \operatorname{Radiant}(|\Delta(\mathcal{C})|)$ there is an integer $t \geq 0$ such that, for every cone $\sigma$ of $\Delta_{t}, g$ is linear over $\sigma$. In symbols, $g \in \operatorname{SF}\left(\mathbf{Z}^{n}, \Delta_{t}\right)$.

Proof. Let the pieces of $g$ be given by the list $\left\{l_{1}, \ldots, l_{z}\right\}$ of homogeneous linear polynomials with integer coefficients. Let $\left\{\mathbf{l}_{1}, \ldots, \mathbf{l}_{z}\right\}$ be their associated bra vectors in $\mathbf{Z}^{n}$. For each $i, j \in\{1, \ldots, z\}$ let $\mathbf{l}_{i j}=\mathbf{l}_{i}-\mathbf{l}_{j}$.

Recalling the definition of lexicographic order given in Section 1, by Lemmas 3.5 and 3.6 there exists an integer $t^{*} \geq 0$ such that, for each $\mathbf{l}_{i j}$, the $\mathbf{l}_{i j}$-temperature of every 2-dimensional cone $\sigma \in \Delta_{t^{*}}$ is $\geq 0$.

Claim: For each cone $\sigma \in \Delta_{t^{*}}$, the polynomials $l_{i}$ are stratified over $\sigma$, in the sense that for some permutation $\omega=\omega(\sigma)$ of the set $\{1, \ldots, z\}$ the simultaneous inequalities $l_{\omega(1)} \leq l_{\omega(2)} \leq \cdots \leq l_{\omega(z)}$ hold all over $\sigma$.

As a matter of fact, if the cone $\sigma$ were a counterexample, then we would have two polynomials $l_{i}$ and $l_{j}$ and points $\mathbf{x}, \mathbf{y} \in \sigma$ such that $l_{i}(\mathbf{x})>l_{j}(\mathbf{x})$ and $l_{i}(\mathbf{y})<l_{j}(\mathbf{y})$. Thus the linear polynomial $l_{i j}=l_{i}-l_{j}$ changes its sign over $\sigma$. Therefore, it is impossible that $l_{i j} \geq 0$ over each 1-dimensional face of the simplicial cone $\sigma$. 
Similarly, we cannot have $l_{i j} \leq 0$ over each 1-dimensional face of $\sigma$. We conclude that the linear polynomial $l_{i j}$ must change its sign over some 2-dimensional face $\tau=\langle\mathbf{v}, \mathbf{w}\rangle$ of $\sigma$. In other words, the $\mathbf{l}_{i j}$-temperature of $\{\mathbf{v}, \mathbf{w}\}$ is $<0$. Since this is impossible in $\Delta_{t^{*}}$, we have reached the desired contradiction to settle our claim.

Since the linear pieces of $g$ are stratified over each cone of $\Delta_{t^{*}}$, it is easy to see that $g$ must be linear over each cone $\sigma \in \Delta_{t^{*}}$.

Lemma 3.8. For each $i=0,1,2, \ldots$ the group $\operatorname{SF}\left(\mathbf{Z}^{n}, \Delta_{i}\right)$ coincides with the subgroup of Radiant $(|\Delta(\mathcal{C})|)$ generated by the set $\left.\mathcal{H}\left(\Delta_{i}\right)\right)$ of Schauder hats, and we have the identity

$$
\operatorname{Radiant}(|\Delta(\mathcal{C})|)=\bigcup_{i} \operatorname{SF}\left(\mathbf{Z}^{n}, \Delta_{i}\right)
$$

The monoid $\left(\operatorname{SF}\left(\mathbf{Z}^{n}, \Delta_{i}\right)\right)^{+}$of $\Delta_{i}$-linear support functions $s \geq 0$ coincides with the sub-monoid of Radiant $(|\Delta(\mathcal{C})|)$ generated by $\mathcal{H}\left(\Delta_{i}\right)$, and we have the identity

$$
(\operatorname{Radiant}(|\Delta(\mathcal{C})|))^{+}=\bigcup_{i}\left(\operatorname{SF}\left(\mathbf{Z}^{n}, \Delta_{i}\right)\right)^{+} .
$$

Proof. By Lemma 3.4, the union of the subgroups $\operatorname{SF}\left(\mathbf{Z}^{n}, \Delta_{i}\right)$ is a subgroup of Radiant $(|\Delta(\mathcal{C})|)$, and the union of the positive cones $\left(\operatorname{SF}\left(\mathbf{Z}^{n}, \Delta_{i}\right)\right)^{+}$is a subset of the positive cone $(\operatorname{Radiant}(|\Delta(\mathcal{C})|))^{+}$. From Lemma 3.7 we see that this union in fact coincides with $(\operatorname{Radiant}(|\Delta(\mathcal{C})|))^{+}$. The desired conclusion now follows from $\operatorname{Radiant}(|\Delta(\mathcal{C})|)$ being generated, as a group, by its positive cone.

Construction of $\lim D(\mathcal{C})$ : Given a non-degenerate abstract simplicial complex $\mathcal{C}$ over $\{1,2, \ldots, n\}$, and its direct system

$$
D(\mathcal{C})=\left\{\varphi_{i}: \mathbf{Z}^{n+i} \rightarrow \mathbf{Z}^{n+i+1} \mid i=0,1, \ldots\right\},
$$

in the light of Lemma 3.3, the general construction of the partially ordered group $U=\lim D(\mathcal{C})$ can be simplified as follows:

C1: For all $0 \leq i<j$, let $\varphi_{i}^{j}: \mathbf{Z}^{n+i} \rightarrow \mathbf{Z}^{n+j}$ be the composite function

$$
\varphi_{j-1} \cdots \varphi_{i+2} \varphi_{i+1} \varphi_{i}
$$

with $\varphi_{i}^{i}$ being the identity over $\mathbf{Z}^{n+i}$.

C2: Over the set $\mathbf{Z}^{\geq n}=\mathbf{Z}^{n} \cup \mathbf{Z}^{n+1} \cup \mathbf{Z}^{n+2} \cup \ldots$, define the equivalence relation $\sim$ by stipulating that, for all $\mathbf{x} \in \mathbf{Z}^{n+i}$ and $\mathbf{y} \in \mathbf{Z}^{n+j}, \mathbf{x} \sim \mathbf{y}$ iff $\varphi_{i}^{\max (i, j)}(\mathbf{x})=$ $\varphi_{j}^{\max (i, j)}(\mathbf{y})$. For every $\mathbf{x} \in \mathbf{Z}^{\geq n}$ let $[\mathbf{x}]$ denote the $\sim$-equivalence class of $\mathbf{x}$.

C3: Define the underlying set of $U$ to be the set $\mathbf{Z}^{\geq n} / \sim$ of $\sim$-equivalence classes. For each $i=0,1, \ldots$, define the map $\varphi_{i}^{\infty}: \mathbf{Z}^{n+i} \rightarrow \mathbf{Z}^{\geq n} / \sim$ by

$$
\varphi_{i}^{\infty}(\mathbf{x})=[\mathbf{x}] .
$$

Note that $\varphi_{i}^{\infty}$ is $1-1$ and $\mathbf{Z} \geq n / \sim=\bigcup_{i} \varphi_{i}^{\infty}\left(\mathbf{Z}^{n+i}\right)$.

C4: For all $[\mathbf{x}],[\mathbf{y}] \in \mathbf{Z}^{\geq n} / \sim$, define $-[\mathbf{x}]=[-\mathbf{x}]$; further, supposing without loss of generality that $\mathbf{x}$ and $\mathbf{y}$ are elements of the same group $\mathbf{Z}^{n+k}$, define $[\mathbf{x}]+[\mathbf{y}]=[\mathbf{x}+\mathbf{y}]$. In this way, the set $\mathbf{Z}^{\geq n} / \sim$ is endowed with the group operations induced by the maps $\varphi_{i}^{\infty}$.

C5: Declare an element $[\mathbf{y}] \in \mathbf{Z}^{\geq n} / \sim$ to be positive iff, letting $j$ be the uniquely determined integer such that $\mathbf{y} \in \mathbf{Z}^{n+j}$, it follows that $\mathbf{y}$ is positive in the 
simplicial group $\mathbf{Z}^{n+j}$, i.e., all coordinates of $\mathbf{y}$ are $\geq 0$. Note that if $\mathbf{y} \sim \mathbf{x}$ and $\mathbf{x} \in \mathbf{Z}^{n+i}$, then also $\mathbf{x}$ is positive in the simplicial group $\mathbf{Z}^{n+i}$.9

Call $U$ the resulting partially ordered abelian group.

Lemma 3.9. (i) $U$ is the limit of the direct system (10) in the category of partially ordered abelian groups, in symbols,

$$
U=\lim _{\rightarrow} D(\mathcal{C}) .
$$

Further, $U$ is a dimension group.

(ii) Each map $\varphi_{i}^{\infty}: \mathbf{Z}^{n+i} \rightarrow U$ is an injective homomorphism sending the positive cone of $\mathbf{Z}^{n+i}$ into the positive cone of $U$, and sending each non-positive element of $\mathbf{Z}^{n+i}$ into a non-positive element of $U$.

(iii) Let the map

$$
\iota: \bigcup_{i} \operatorname{SF}\left(\mathbf{Z}^{n}, \Delta_{i}\right) \rightarrow \lim _{\rightarrow} D(\mathcal{C})
$$

send each $f \in \operatorname{SF}\left(\mathbf{Z}^{n}, \Delta_{i}\right)$ into the $\sim$-equivalence class $\left[\eta_{i}(f)\right]=\varphi_{i}^{\infty}\left(\eta_{i}(f)\right) \in$ $U$. Then $\iota$ is an isomorphism of $\operatorname{Radiant}(|\Delta(\mathcal{C})|)$ onto the dimension group $U=$ $\lim _{\rightarrow} D(\mathcal{C})$.

Proof. (i) This follows from the Effros-Handelman-Shen characterization of dimension groups 6], [11.

(ii) Immediate from Lemma 3.3

(iii) Using Lemma 3.8 we have Radiant $(|\Delta(\mathcal{C})|)=\bigcup \operatorname{SF}\left(\mathbf{Z}^{n}, \Delta_{i}\right)$. By Lemma 3.9, $\iota$ is a 1-1 homomorphism into $U$, sending each function $0 \leq p \in \operatorname{SF}\left(\mathbf{Z}^{n}, \Delta_{i}\right)$ into a positive element of $U$, and sending each non-positive function $q \in \mathrm{SF}\left(\mathbf{Z}^{n}, \Delta_{i}\right)$ into a non-positive element of $U$.

To see that $\iota$ is surjective, let $[\mathbf{x}]$ be an element of the positive cone of $U$. For some integer $i \geq 0$ we can write $\mathbf{x} \in \mathbf{Z}^{n+i}$ and $[\mathbf{x}]=\varphi_{i}^{\infty}(\mathbf{x})$. In the simplicial group $\mathbf{Z}^{n+i}, \mathbf{x}$ is a positive linear combination of the atoms $\mathbf{e}_{1}, \ldots, \mathbf{e}_{n+i}$, with integer coefficients $\geq 0$. By Proposition 2.1, its corresponding image $g=\eta_{i}^{-1}(\mathbf{x})$ is a function in $\operatorname{SF}\left(\mathbf{Z}^{n}, \Delta_{i}\right)$ which can be uniquely written as a linear combination of the Schauder hats of $\Delta_{i}$ with the same coefficients. We now have

$$
\iota(g)=\varphi_{i}^{\infty}\left(\eta_{i}(g)\right)=\varphi_{i}^{\infty}(\mathbf{x})=[\mathbf{x}] .
$$

Since $U^{+}-U^{+}=U$, we get that $\iota$ is surjective.

This completes the proof of Theorem 3.1

\section{4. $\ell$-GROUP PRESENTATIONS AND $\ell$-EQUIVALENCE}

An abelian lattice ordered group (for short, an $\ell$-group) $G$ is an abelian group that is also a lattice satisfying the equations $x+(y \vee z)=(x+y) \vee(x+z)$ and $x+(y \wedge z)=(x+y) \wedge(x+z)$. In agreement with the usual notation for partially ordered abelian groups, the positive cone $G^{+}$is defined by

$$
G^{+}=\{z \in G \mid z \geq 0\} .
$$

For every $X \subseteq \mathbf{R}^{n}$ the group $\operatorname{Radiant}(X)$ of radiant functions over $X$ with pointwise max-min operations is an example of an $\ell$-group.

\footnotetext{
${ }^{9}$ This is so because each map $\varphi_{i}$ is strong (Lemma 3.3 (iv)).
} 
Given a nonempty set $Y$, we denote by $F_{Y}$ the free $\ell$-group on (the free generating set) $Y$.

Proposition 4.1. Let $Y=\{1, \ldots, n\}$. Then the free $\ell$-group $F_{Y}$ can be identified with the $\ell$-group $\operatorname{Radiant}\left(\mathbf{R}^{n}\right)$ of all radiant functions $f: \mathbf{R}^{n} \rightarrow \mathbf{R}$, with the free generating set given by the projection functions $\pi_{i}: \mathbf{R}^{n} \rightarrow \mathbf{R}$, where $\pi_{i}\left(x_{1}, \ldots, x_{n}\right)=x_{i}$ for all $\left(x_{1}, \ldots, x_{n}\right) \in \mathbf{R}^{n}$ and $i=1, \ldots, n$. In symbols,

$$
F_{Y}=F_{\left\{\pi_{1}, \ldots, \pi_{n}\right\}}=F_{n}=\operatorname{Radiant}\left(\mathbf{R}^{n}\right) .
$$

Under this identification, every element $f \in F_{n}$ can be written in the form

$$
f=\bigvee_{i} \bigwedge_{j}\left\{l_{i j} \mid i \in I, j \in J\right\}
$$

where $I$ and $J$ are finite sets, and each $l_{i j}$ is a homogeneous linear polynomial over $\mathbf{Z}^{n}$ with integer coefficients.

Proof. Well known [9, 2], 1].

A finitely presented $\ell$-group $G$ with generators $g_{1}, \ldots, g_{m}$ and defining relations $a_{1}=b_{1}, \ldots, a_{t}=b_{t}$ (where all $a$ 's and $b$ 's are elements of $F_{\left\{g_{1}, \ldots, g_{m}\right\}}$ ) is the quotient of $F_{\left\{g_{1}, \ldots, g_{m}\right\}}$ by the congruence generated by $a_{1}=b_{1}, \ldots, a_{t}=b_{t}$. If $\alpha_{i}$ and $\beta_{i} \quad(i=1, \ldots, t)$ are $\ell$-group terms in the variable symbols $X_{1}, \ldots, X_{m}$ representing the elements $a_{i}$ and $b_{i}$, we say that the string of symbols

$$
\wp=\left[\left[X_{1} \ldots, X_{m} ; \alpha_{1}=\beta_{1}, \ldots, \alpha_{t}=\beta_{t}\right]\right]
$$

is a presentation of $G$.

Two sets $E_{1} \subseteq \mathbf{R}^{p}$ and $E_{2} \subseteq \mathbf{R}^{q}$ are $\ell$-equivalen 10 iff there exist functions

$$
f_{1}, \ldots, f_{q} \in \operatorname{Radiant}\left(\mathbf{R}^{p}\right) \text { and } g_{1}, \ldots, g_{p} \in \operatorname{Radiant}\left(\mathbf{R}^{q}\right)
$$

such that, defining $\mathbf{f}: \mathbf{R}^{p} \rightarrow \mathbf{R}^{q}$ and $\mathbf{g}: \mathbf{R}^{q} \rightarrow \mathbf{R}^{p}$ by

$$
\mathbf{f}(\mathbf{y})=\left(f_{1}(\mathbf{y}), \ldots, f_{q}(\mathbf{y})\right) \text { and } \mathbf{g}(\mathbf{z})=\left(g_{1}(\mathbf{z}), \ldots, g_{p}(\mathbf{z})\right),
$$

we have the identities $\mathbf{g}(\mathbf{f}(\mathbf{y}))=\mathbf{y}$ and $\mathbf{f}(\mathbf{g}(\mathbf{z}))=\mathbf{z}$, for all $\mathbf{y} \in E_{1}$ and $\mathbf{z} \in E_{2}$. If necessary, we may specify that $E_{1}$ and $E_{2}$ are $\ell$-equivalent via the maps $\mathbf{f}$ and $\mathbf{g}$.

Following tradition, for every element $x \in G$ we let $|x|=x \vee-x$.

Proposition 4.2. (i) Suppose $\wp=\left[\left[X_{1}, \ldots, X_{m} ; \alpha_{1}=\beta_{1}, \ldots, \alpha_{t}=\beta_{t}\right]\right]$ is a presentation of an $\ell$-group $G$. Let the $\ell$-group term $\gamma=\gamma_{\wp}=\gamma\left(X_{1} \ldots, X_{m}\right)$ be given by $\gamma=\left|\alpha_{1}-\beta_{1}\right| \vee \ldots \vee\left|\alpha_{t}-\beta_{t}\right|$. Let $Z_{\wp}=f_{\gamma}^{-1}(0)$ be the zero set of the radiant function $f_{\gamma}$ represented by $\gamma$ in $F_{m}$ as in (11). Then $G$ is also presented by $\wp^{\prime}=\left[\left[X_{1} \ldots, X_{m} ; \gamma=0\right]\right]$, and $G \cong \operatorname{Radiant}\left(Z_{\wp}\right)$.

(ii) Suppose $\wp_{1}=\left[\left[Y_{1} \ldots, Y_{p} ; \gamma_{1}=0\right]\right]$ and $\wp_{2}=\left[\left[Z_{1} \ldots, Z_{q} ; \gamma_{2}=0\right]\right]$ are presentations of the $\ell$-groups $G_{1}$ and $G_{2}$, with their zero sets $Z_{\wp_{1}} \subseteq \mathbf{R}^{p}$ and $Z_{\wp_{2}} \subseteq \mathbf{R}^{q}$. Then $G_{1} \cong G_{2}$ iff $Z_{\wp_{1}}$ and $Z_{\wp_{2}}$ are $\ell$-equivalent.

Proof. Both statements are well-known consequences of the Baker-Beynon duality ([9. Lemma 0 and references therein]).

\footnotetext{
${ }^{10}$ To be understood as an abbreviation of "piecewise homogeneous linearly equivalent (with integer coefficients)".
} 
Proposition 4.3. There is a Turing machine $\Theta_{1}$ which, having as input the presentation $\wp=\left[\left[X_{1} \ldots, X_{m} ; \alpha_{1}=\beta_{1}, \ldots, \alpha_{t}=\beta_{t}\right]\right]$ of an $\ell$-group $G$, outputs a non-singular fan $\Delta_{\wp}$ in $\mathbf{R}^{m}$ such that

$$
G \cong \operatorname{Radiant}\left(\left|\Delta_{\wp}\right|\right)
$$

and the number $n$ of 1-dimensional cones in $\Delta_{\wp}$ satisfies the inequality $n \geq m$. Specifically, $\Theta_{1}$ outputs the finite sequence of sets of primitive generating vectors of every cone in $\Delta_{\wp}$.

Proof. As in the above proposition, we first transform $\wp$ into the equivalent presentation

$$
\wp^{\prime}=\left[\left[X_{1} \ldots, X_{m} ; \gamma=0\right]\right] .
$$

Trivially, $\wp^{\prime}$ can be effectively computed from $\wp$. Again using standard manipulations and recalling (11)-(12), we can rewrite $\gamma$ in the equivalent form

$$
\bigwedge_{i=1}^{p} \bigvee_{j=1}^{q} \psi_{i j}
$$

where each $\psi_{i j}$ is (a term over the variables $X_{1} \ldots, X_{m}$ in the language of groups, representing) a homogeneous linear polynomial over $\mathbf{R}^{m}$ with integer coefficients. Each variable symbol $X_{i}$ represents the $i$ th projection function $\pi_{i}$ over $\mathbf{R}^{m}$. We can safely assume that the $\ell$-group term 0 is among the $\psi_{i j}$ 's. Let $\Lambda$ be the following list of $\ell$-group terms:

$$
\Lambda=\left\{X_{1} \ldots, X_{m}, \psi_{11}, \ldots, \psi_{p q}\right\} .
$$

For each permutation $\omega$ of the set $\{1, \ldots, p\} \times\{1, \ldots, q\}$, and for each function $\epsilon$ : $\{1, \ldots, m\} \rightarrow\{1,-1\}$, let $\sigma_{\omega \epsilon}$ be set of solutions in $\mathbf{R}^{m}$ of the system of linear inequalities in the "unknowns" $X_{1} \ldots, X_{m}$

$$
\left\{\begin{array}{l}
\psi_{\omega(11)} \leq \psi_{\omega(12)} \leq \cdots \leq \psi_{\omega(1 q)} \leq \psi_{\omega(21)} \leq \psi_{\omega(22)} \leq \cdots \leq \psi_{\omega(p q)}, \\
\epsilon(1) X_{1} \geq 0 \\
\vdots \\
\epsilon(m) X_{m} \geq 0 .
\end{array}\right.
$$

A moment's reflection shows that each $\sigma_{\omega \epsilon}$ is a rational convex polyhedral cone and is pointed (in the sense that no line is contained in $\sigma_{\omega \epsilon}$ ). Further, the collection of all faces of all cones $\sigma_{\omega \epsilon}$ forms a complex $\widetilde{\Gamma_{\wp}}$ of rational convex pointed polyhedral cones, whose support is $\mathbf{R}^{m}$. There is a Turing machine which for input $\wp$ computes the set of primitive generating vectors in $\mathbf{Z}^{m}$ for every cone of $\widetilde{\Gamma_{\wp}}$. Indeed, the above system (14) is effectively constructed from $\wp$, and the cones in $\mathbf{R}^{m}$ solving (14) can be effectively presented by their primitive generating vectors [10] Lemma 5 and Theorem 6].

Recalling (11), once interpreted in the free $\ell$-group $F_{m}$, the $\ell$-term $\gamma$ represents a radiant function $f_{\gamma}: \mathbf{R}^{m} \rightarrow \mathbf{R}$, whose linear pieces are given by (some of) the polynomials $f_{\psi}$ represented by the $\psi$ 's. Over each cone $\sigma_{\omega \epsilon}, f_{\gamma}$ is linear and coincides with some $f_{\psi}$. Let $\Gamma_{\wp}$ denote the subcomplex of $\widetilde{\Gamma_{\wp}}$ given by those cones $\sigma$ of $\widetilde{\Gamma_{\wp}}$ such that $f_{\gamma}$ vanishes over $\sigma$. It follows that the zero set $Z_{\wp}=f_{\gamma}^{-1}(0)$ of $f_{\gamma}$ 
coincides with the support of $\Gamma_{\wp}$. From Proposition 4.2 it follows that

$$
G \cong \operatorname{Radiant}\left(\left|\Gamma_{\wp}\right|\right) \text {. }
$$

To conclude the proof we must effectively compute (the primitive generating vectors of all cones of) a non-singular fan $\Delta_{\wp}$ with the same support of $\Gamma_{\wp}$, in such a way that every cone of $\Gamma_{\wp}$ is the set-theoretic union of cones of $\Delta_{\wp}$. To this purpose, for all suitably large integers $l$, direct inspection on the desingularization algorithms of [8, Theorem 8.5, p. 253] or [18 p. 23] yields such an "effective resolution of singularities" $\Delta_{\wp}$ of $\Gamma_{\wp}$, having $l 1$-dimensional cones. Pick the smallest such integer $n$ satisfying $n \geq m$.

For any presentation $\wp=\left[\left[X_{1} \ldots, X_{m} ; \alpha_{1}=\beta_{1}, \ldots, \alpha_{t}=\beta_{t}\right]\right]$ of an $\ell$-group $G$ we shall denote by $\Delta_{\wp}$ the output of the Turing machine $\Theta_{1}$ of Proposition 4.3 for input $\wp$.

Lemma 4.4. Let $\wp$ be the presentation of an $\ell$-group $G$. Then $G$ has infinitely many maximal $\ell$-ideals iff $\Delta_{\wp}$ contains (the primitive generating vectors of) some 2 -dimensional cone. Thus, the property of $\wp$ being a presentation of an $\ell$-group having infinitely many maximal $\ell$-ideals is decidable by some Turing machine $\Psi$.

Proof. Adopting the notation of the proof of Proposition 4.3 and recalling (15), let us identify $G$ with the $\ell$-group of restrictions to the zero set $Z_{\wp}=f_{\gamma_{\wp}}^{-1}(0)=\left|\Delta_{\wp}\right|$ of all functions in the free $m$-generated $\ell$-group $F_{m}$; in symbols,

$$
G=\operatorname{Radiant}\left(Z_{\wp}\right)=\operatorname{Radiant}\left(\left|\Delta_{\wp}\right|\right) .
$$

If $\Delta_{\wp}$ does not contain any two-dimensional cone, then $\left|\Delta_{\wp}\right|$ is the union of a finite number of 1-dimensional cones $\rho_{1}, \ldots, \rho_{k}$. It follows from (16) that $G$ is the $k$-fold cartesian product of the additive group of integers with the natural order. In other words, $G$ is simplicial, whence $G$ has only finitely many maximal $\ell$-ideals.

If, on the other hand, $\Delta_{\wp}$ possesses at least one 2-dimensional cone $\sigma=\langle\mathbf{v}, \mathbf{w}\rangle$, then for each 1-dimensional rational cone $\rho \subseteq \sigma$, the restriction to $\rho$ of all functions of $G$ yields an $\ell$-homomorphism $\theta_{\rho}$ of $G$ onto $\mathbf{Z}$. The kernel of $\theta_{\rho}$ is a maximal $\ell$ ideal of $G$. Since different $\rho$ 's give different maximal $\ell$-ideals, $G$ has infinitely many maximal $\ell$-ideals. By direct inspection on the output string $\Delta_{\wp}$, of the Turing machine $\Theta_{1}$, we can decide whether $\Delta_{\wp}$ contains a 2-dimensional cone.

Theorem 4.5. There is a Turing machine $\Theta_{2}$ which, given the presentation $\wp$ of an $\ell$-group $G$, proceeds as follows:

- If $\wp$ is the presentation of an $\ell$-group having finitely many maximal $\ell$-ideals, $\Theta_{2}$ outputs the number 0 .

- If $\wp$ is the presentation of an $\ell$-group having infinitely many maximal $\ell$ ideals, $\Theta_{2}$ outputs an abstract simplicial complex $\mathcal{C}_{\wp}$ such that, letting $\Delta\left(\mathcal{C}_{\wp}\right)$ be the initial non-singular fan of $\mathcal{C}_{\wp}$, we have the isomorphism

$$
G \cong \operatorname{Radiant}\left(\left|\Delta\left(\mathcal{C}_{\wp}\right)\right|\right) .
$$

Proof. Initially, $\Theta_{2}$ waits for the output $\Delta_{\wp}$ of the Turing machine $\Theta_{1}$ of Proposition 4.3. Let $\rho_{1}=\left\langle\mathbf{v}_{1}\right\rangle, \ldots, \rho_{n}=\left\langle\mathbf{v}_{n}\right\rangle$ be the 1-dimensional cones of the non-singular fan $\Delta_{\wp}$ in $\mathbf{Z}^{m}$. The problem of whether or not the $\ell$-group presented by $\wp$ has infinitely many maximal $\ell$-ideals can be decided by the Turing machine $\Psi$ of Lemma 4.4 by direct inspection on $\Delta_{\wp}$. 
If there are no 2-dimensional cones in $\Delta_{\wp}$, then $\Theta_{2}$ outputs the 0 symbol and halts.

Otherwise, if there exists a 2-dimensional cone in $\Delta_{\wp}$, then machine $\Theta_{2}$ extracts from $\Delta_{\wp}$ the non-degenerate abstract simplicial complex $\mathcal{C}=\mathcal{C}_{\wp}$ over $\{1, \ldots, n\}$ as follows: a set $S \subseteq\{1, \ldots, n\}$ is in $\mathcal{C}$ iff $\left\{\mathbf{v}_{j} \mid j \in S\right\}$ is the set of primitive generating vectors of some cone in $\Delta_{\wp}$. The map $\wp \mapsto \mathcal{C}_{\wp}$ is Turing-computable. Now $\Theta_{2}$ transforms $\mathcal{C}_{\wp}$ into the new fan $\Delta\left(\mathcal{C}_{\wp}\right)$ in $\mathbf{R}^{n}$ obtained by mapping each index $i=1, \ldots, n$ into the basis vectors $\mathbf{e}_{1}, \ldots, \mathbf{e}_{n}$ of $\mathbf{Z}^{n}$, as is done in Section 1 Trivially, $\Delta\left(\mathcal{C}_{\wp}\right)$ is non-singular. We shall now prove

Claim: The supports $\left|\Delta_{\wp}\right| \subseteq \mathbf{R}^{m}$ and $\left|\Delta\left(\mathcal{C}_{\wp}\right)\right| \subseteq \mathbf{R}^{n}$ are $\ell$-equivalent.

We must exhibit radiant functions

$$
f_{1}, \ldots, f_{n}: \mathbf{R}^{m} \rightarrow \mathbf{R} \text { and } g_{1}, \ldots, g_{m}: \mathbf{R}^{n} \rightarrow \mathbf{R}
$$

such that $\mathbf{g}(\mathbf{f}(\mathbf{x}))=\mathbf{x}$ for all $\mathbf{x} \in\left|\Delta_{\wp}\right|$, and $\mathbf{f}(\mathbf{g}(\mathbf{y}))=\mathbf{y}$ for all $\mathbf{y} \in\left|\Delta\left(\mathcal{C}_{\wp}\right)\right|$. To this purpose, for every cone $\sigma=\left\langle\mathbf{v}_{\omega(1)}, \ldots, \mathbf{v}_{\omega(k)}\right\rangle \in \Delta_{\wp}$, with $1 \leq \omega(1)<$ $\cdots<\omega(k) \leq n$, we shall map $\sigma$ one-to-one onto the corresponding cone $\sigma^{\prime}=$ $\left\langle\mathbf{e}_{\omega(1)}^{\prime}, \ldots, \mathbf{e}_{\omega(k)}^{\prime}\right\rangle \subseteq \mathbf{Z}^{n}$ via a linear transformation with integer coefficients whose inverse also has integer coefficients. Since by Proposition 4.3, $k \leq m \leq n$, then the non-singularity of $\Delta_{\wp}$ yields a basis in $\mathbf{Z}^{m}$ having the form

$$
\mathbf{w}_{1}=\mathbf{v}_{\omega(1)}, \ldots, \mathbf{w}_{k}=\mathbf{v}_{\omega(k)}, \mathbf{w}_{k+1}, \ldots, \mathbf{w}_{m} .
$$

Let $M$ be the $m \times m$ unimodular integer matrix whose $\omega(j)$ th column coincides with the vector $\mathbf{w}_{j}$, for each $j=1, \ldots, m$. The inverse matrix $N=M^{-1}$ sends each of $\mathbf{w}_{1}, \ldots, \mathbf{w}_{m}$ into its corresponding basis vector $\mathbf{e}_{1}, \ldots, \mathbf{e}_{m}$ of $\mathbf{Z}^{m}$, whence, in particular, $N \mathbf{v}_{\omega(j)}=\mathbf{e}_{j}(j=1, \ldots, k)$. Let the $n \times m$ matrix $A$ be defined as follows:

- for each $k<j \leq m$ the $j$ th column of $A$ is zero;

- for each $1 \leq j \leq k$ the $j$ th column of $A$ is zero except at its $\omega(j)$ th entry.

Let $B=B_{\sigma}=A N$. Then for each $j=1, \ldots, k, B_{\sigma} \mathbf{v}_{\omega(j)}=A \mathbf{e}_{j}=\mathbf{e}_{\omega(j)}^{\prime} \in \mathbf{Z}^{n}$.

We shall show that the linear transformation

$$
B_{\sigma}:\left\langle\mathbf{v}_{\omega(1)}, \ldots, \mathbf{v}_{\omega(k)}\right\rangle \cong\left\langle\mathbf{e}_{\omega(1)}^{\prime}, \ldots, \mathbf{e}_{\omega(k)}^{\prime}\right\rangle
$$

has an inverse, also with integer coefficients. As a matter of fact, let $C=C_{\sigma}$ be the $m \times n$ integer matrix whose $\omega(j)$ th column $(j=1, \ldots, k)$ coincides with the vector $\mathbf{v}_{\omega(j)}$, and whose remaining columns are zero. Then $C_{\sigma}$ sends the generating vectors of the cone $\sigma^{\prime}=\left\langle\mathbf{e}_{\omega(1)}^{\prime}, \ldots, \mathbf{e}_{\omega(k)}^{\prime}\right\rangle \subseteq \mathbf{Z}^{n}$ corresponding to $\sigma$ one-to-one onto the generating vectors of $\sigma$, and we can write

$$
C_{\sigma}:\left\langle\mathbf{e}_{\omega(1)}^{\prime}, \ldots, \mathbf{e}_{\omega(k)}^{\prime}\right\rangle \cong\left\langle\mathbf{v}_{\omega(1)}, \ldots, \mathbf{v}_{\omega(k)}\right\rangle
$$

Thus, $C_{\sigma}$ is a one-to-one linear map, with integer coefficients, of $\sigma^{\prime}$ onto $\sigma=\left\langle\mathbf{v}_{\omega(1)}\right.$, $\left.\ldots, \mathbf{v}_{\omega(k)}\right\rangle \subseteq \mathbf{Z}^{m}$. Trivially, the maps $B_{\sigma}$ and $C_{\sigma}$ are inverses of each other.

To conclude the proof of our claim, let $\sigma$ range over all cones of $\Delta_{\wp}$. Define the (patching) function

$$
\mathbf{p}=\left(p_{1}, \ldots, p_{n}\right):\left|\Delta_{\wp}\right| \rightarrow\left|\Delta\left(\mathcal{C}_{\wp}\right)\right|
$$

as the function which over each $\sigma$ equals $B_{\sigma}$. This is a well-defined one-to-one map of $\left|\Delta_{\wp}\right|$ onto $\left|\Delta\left(\mathcal{C}_{\wp}\right)\right|$, and each $p_{j}:\left|\Delta_{\wp}\right| \rightarrow \mathbf{R}$ is by construction a $\Delta_{\wp}$-linear support function. Arguing as in the proof of Proposition 2.3 and extending $\Delta_{\wp}$ to 
a non-singular fan $\Delta_{\wp}^{\diamond}$ over the whole of $\mathbf{R}^{m}$, we see that, for each $j=1, \ldots, n, p_{j}$ is the restriction to $\left|\Delta_{\wp}\right|$ of some radiant function $f_{j}: \mathbf{R}^{m} \rightarrow \mathbf{R}$.

Symmetrically, letting $\sigma^{\prime}$ range over all cones of $\Delta\left(\mathcal{C}_{\wp}\right)$, we define by patching the function

$$
\mathbf{q}=\left(q_{1}, \ldots, q_{m}\right):\left|\Delta\left(\mathcal{C}_{\wp}\right)\right| \rightarrow\left|\Delta_{\wp}\right|,
$$

by stipulating that $\mathbf{q}$ coincides with $C_{\sigma^{\prime}}$ over each cone $\sigma^{\prime}$. Each $q_{i}$ is a $\Delta\left(\mathcal{C}_{\wp}\right)$ linear support function and is the restriction to $\left|\Delta\left(\mathcal{C}_{\wp}\right)\right|$ of some radiant function $g_{i}: \mathbf{R}^{n} \rightarrow \mathbf{R}$. Furthermore, $\mathbf{q}$ maps $\left|\Delta\left(\mathcal{C}_{\wp}\right)\right|$ one-to-one onto $\left|\Delta_{\wp}\right|$. Since $B_{\sigma}$ and $C_{\sigma}$ are inverses of each other, we have settled our claim.

Using Proposition 4.2, we get

$$
\operatorname{Radiant}\left(\left|\Delta_{\wp}\right|\right) \cong \operatorname{Radiant}\left(\left|\Delta\left(\mathcal{C}_{\wp}\right)\right|\right) \text {. }
$$

The desired conclusion now follows from (13).

\section{Conclusion of the proof of the main theorem}

End of proof of (i). By Theorems 4.5 and 3.1, together with Lemma 4.4, there is a Turing machine $\Theta_{3}$ which, having as input a pair of presentations $\left(\wp, \wp^{\prime}\right)$, in case both $\wp$ and $\wp^{\prime}$ are presentations of $\ell$-groups with infinitely many maximal $\ell$-ideals outputs a pair of abstract simplicial complexes $\left(\mathcal{C}(\wp), \mathcal{C}\left(\wp^{\prime}\right)\right)$ having the following property: $\wp$ and $\wp^{\prime}$ are presentations of isomorphic $\ell$-groups iff $\lim _{\rightarrow} D((\mathcal{C}(\wp)) \cong$ $\lim D\left(\left(\mathcal{C}\left(\wp^{\prime}\right)\right) .11\right.$ By way of contradiction, suppose the $C^{*}$-equivalence problem for non-degenerate abstract simplicial complexes $\mathcal{C}$ and $\mathcal{C}^{\prime}$ is Turing-decidable. Using the Turing machines $\Psi$ and $\Theta_{3}$, we have an effective procedure to decide if $\wp$ and $\wp^{\prime}$ are presentations of isomorphic $\ell$-groups with infinitely many $\ell$-ideals.

From Lemma 4.4 one easily obtains an effective procedure to decide if $\wp$ and $\wp^{\prime}$ are presentations of isomorphic $\ell$-groups with finitely many $\ell$-ideals.

Therefore, there is an effective procedure to decide if $\wp$ and $\wp^{\prime}$ are presentations of isomorphic $\ell$-groups.

Now from Markov's celebrated undecidability theorem for the piecewise linear homeomorphism for compact polyhedra (9] and references therein), one obtains as a corollary that the isomorphism problem for finite presentations of $\ell$-groups is Turing-undecidable [9, Theorem A (2), p. 53]. We have reached the desired contradiction, and the proof of Theorem 1.1(i) is complete.

Proof of (ii). We first consider the following problem:

Assume we are given a pair of non-degenerate abstract simplicial complexes $\mathcal{C}$ and $\mathcal{C}^{\prime}$, respectively over $\{1, \ldots, n\}$ and $\left\{1, \ldots, n^{\prime}\right\}$, together with $\ell$-group terms

$$
\zeta_{1}\left(X_{1}, \ldots, X_{n}\right), \ldots, \zeta_{n^{\prime}}\left(X_{1}, \ldots, X_{n}\right)
$$

and

$$
\theta_{1}\left(Y_{1}, \ldots, Y_{n^{\prime}}\right), \ldots, \theta_{n}\left(Y_{1}, \ldots, Y_{n^{\prime}}\right)
$$

Question: Are the two sets $|(\Delta(\mathcal{C}))|$ and $\left|\left(\Delta\left(\mathcal{C}^{\prime}\right)\right)\right|$-equivalent via the maps $\mathbf{f}=\left(f_{1}, \ldots, f_{n^{\prime}}\right): \mathbf{R}^{n} \rightarrow \mathbf{R}^{n^{\prime}}$ and $\mathbf{g}=\left(g_{1}, \ldots, g_{n}\right): \mathbf{R}^{n^{\prime}} \rightarrow \mathbf{R}^{n}$ represented by $\left(\zeta_{1}, \ldots, \zeta_{n^{\prime}}\right)$ and $\left(\theta_{1}, \ldots, \theta_{n}\right)$ ?

Claim: The above problem is Turing-decidable.

\footnotetext{
${ }^{11}$ And $\Theta_{3}$ outputs the symbol 0 , otherwise.
} 
As a matter of fact, let the functions $p: \mathbf{R}^{n} \rightarrow \mathbf{R}$ and $q: \mathbf{R}^{n^{\prime}} \rightarrow \mathbf{R}$ be defined by $p(\mathbf{x})=|\mathbf{x}-\mathbf{g}(\mathbf{f}(\mathbf{x}))|$ and $q(\mathbf{y})=|\mathbf{y}-\mathbf{f}(\mathbf{g}(\mathbf{y}))|$. Let

$$
Q=\left\{\mathbf{x} \in[-1,1]^{n} \mid \text { some coordinate of } \mathbf{x} \text { is equal to } \pm 1\right\} .
$$

Let $p^{\square}$ be the restriction of $p$ to $Q \cap|\Delta(\mathcal{C})|$.

The maximum of $p^{\square}$ is attained at a point of $Q \cap|\Delta(\mathcal{C})|$ with rational coordinates whose least common denominator $d$ is bounded by some Turing-computable function (given once and for all) of the number of occurrences of variables in the terms $\zeta_{1}, \ldots, \zeta_{n^{\prime}}$ and $\theta_{1}, \ldots, \theta_{n}$, together with the coordinates of the primitive generating vectors of all cones of $\Delta(\mathcal{C})$ and $\Delta\left(\mathcal{C}^{\prime}\right)$. Compare with [14. The rational points in $Q \cap|\Delta(\mathcal{C})|$ whose least common denominator does not exceed this upper bound are only finitely many, and can be effectively listed. Thus by evaluating $p^{\square}$ at all such points one can effectively decide whether $p^{\square}$ is constantly equal to zero over $Q \cap|\Delta(\mathcal{C})|$, or equivalently, decide whether $\mathbf{x}=\mathbf{g}(\mathbf{f}(\mathbf{x}))$ over $|\Delta(\mathcal{C})|$. One can similarly decide, in a finite number of steps, whether $\mathbf{y}=\mathbf{f}(\mathbf{g}(\mathbf{y}))$ over all of $\left|\Delta\left(\mathcal{C}^{\prime}\right)\right|$. The sets $|\Delta(\mathcal{C})|$ and $\left|\Delta\left(\mathcal{C}^{\prime}\right)\right|$ are piecewise linear homogeneous equivalent iff both checks are positive. The claim is settled.

We conclude the proof of part (ii) of the main theorem as follows: Let the Turing machine $\Omega$ enumerate all quadruples

$$
\mathcal{Q}=\left(\mathcal{C}, \mathcal{C}^{\prime},\left(\zeta_{1}, \ldots, \zeta_{n^{\prime}}\right),\left(\theta_{1}, \ldots, \theta_{n}\right)\right),
$$

where

- $\mathcal{C}$ and $\mathcal{C}^{\prime}$ are abstract simplicial complexes, over $\{1,2, \ldots, n\}$ and $\{1,2, \ldots$, $\left.n^{\prime}\right\}$, respectively,

- $\left(\zeta_{1}\left(X_{1}, \ldots, X_{n}\right), \ldots, \zeta_{n^{\prime}}\left(X_{1}, \ldots, X_{n}\right)\right)$ is an $n^{\prime}$-tuple of $\ell$-group terms in the variables $X_{1}, \ldots, X_{n}$, and

- $\left(\theta_{1}\left(Y_{1}, \ldots, Y_{n^{\prime}}\right), \ldots, \theta_{n}\left(Y_{1}, \ldots, Y_{n^{\prime}}\right)\right)$ is an $n$-tuple of $\ell$-group terms in the variables $Y_{1}, \ldots, Y_{n^{\prime}}$.

Letting $\mathbf{f}: \mathbf{R}^{n} \rightarrow \mathbf{R}^{n^{\prime}}$ and $\mathbf{g}: \mathbf{R}^{n^{\prime}} \rightarrow \mathbf{R}^{n}$ be their corresponding maps, one decides, as in the above claim, whether $|\Delta(\mathcal{C})|$ and $\left|\Delta\left(\mathcal{C}^{\prime}\right)\right|$ are $\ell$-equivalent via $\mathbf{f}$ and $\mathbf{g}$. Whenever this is the case, $\left(\mathcal{C}, \mathcal{C}^{\prime}\right)$ is added to the set of $C^{*}$-equivalent pairs; as a matter of fact, by Theorem 3.1 and Proposition $4.2 \mathcal{C}$ is $C^{*}$-equivalent to $\mathcal{C}^{\prime}$ iff the $\ell$-groups $\operatorname{Radiant}(\Delta(\mathcal{C}))$ and $\operatorname{Radiant}\left(\Delta\left(\mathcal{C}^{\prime}\right)\right)$ are isomorphic, iff $|(\Delta(\mathcal{C}))|$ and $\left|\left(\Delta\left(\mathcal{C}^{\prime}\right)\right)\right|$ are piecewise linear homogeneous equivalent.

After examining the quadruple $\mathcal{Q}$, our machine $\Omega$ proceeds to the examination of the next quadruple. We have proved that the set of $C^{*}$-equivalent pairs $\left(\mathcal{C}, \mathcal{C}^{\prime}\right)$ is recursively enumerable (yet undecidable, by part (i)). This completes the proof of part (ii) of the main theorem.

The proof of Theorem 1.1 is complete.

Remarks. 1. From the proof of Theorems 4.5 and 3.1, one has a constructive proof that every finitely presented $\ell$-group $G$ is ultrasimplicial (i.e., $G$ is the limit of a direct system of simplicial groups with order-preserving 1-1 homomorphisms $\varphi_{i}$ ). Furthermore, by Lemma 3.3 each $\varphi_{i}$ is strong with respect to the order relation, and its image is pure. Compare with [15. Theorem 2.1] (also see [16]), and with the general, albeit non-constructive, result [12], to the effect that every $\ell$-group is ultrasimplicial.

2. In general, it is hard to decide whether the dimension group arising as the limit of a system of simplicial groups and positive integer matrices is lattice-ordered [7]. By 
Theorem [3.1, all dimension groups arising from non-degenerate abstract simplicial complexes are automatically lattice-ordered.

\section{GÖDEL-INCOMPLETE STABLE ISOMORPHISM PROBLEMS}

In this final section we reformulate our main theorem in the language of AFalgebras, using Elliott's classification theory [6], [11]. Recall that an AF-algebra $A$ is a separable $C^{*}$-algebra such that for every $\epsilon>0$ and every finite subset $B \subseteq A$ there are a finite-dimensional ${ }^{*}$-subalgebra $A^{\prime}$ of $A$ and a finite subset $B^{\prime} \subseteq A^{\prime}$ approximating $B$ up to error $\epsilon$. If, in addition, $A$ is isomorphic to its tensor product with the compact operators on a separable Hilbert space, then $A$ is said to be stable.

Grothendieck's $K_{0}$-functor transforms every stable AF-algebra $A$ into a countable dimension group $\left(K_{0}(A), K_{0}(A)^{+}\right)$, in such a way that isomorphism classes of stable AF-algebras are in one-to-one correspondence with isomorphism classes of countable dimension groups. It follows from Theorem 3.1 that two abstract simplicial complexes $\mathcal{C}, \mathcal{C}^{\prime}$ are $C^{*}$-equivalent iff the stable $\mathrm{AF}$-algebras $A(\mathcal{C})$ and $A\left(\mathcal{C}^{\prime}\right)$ associated to the dimension groups $\lim D(\mathcal{C})$ and $\lim D\left(\mathcal{C}^{\prime}\right)$ are isomorphic. The direct system (1) is known as a (Bratteli) diagram for $A \overrightarrow{12}$

In AF-algebraic terms our main result can be read as the Gödel-incompleteness of the $C^{*}$-equivalence problem for a certain class of simply described AF-algebras as follows:

Theorem 6.1. (i) There is no algorithm to decide isomorphism of stable AFalgebras arising from non-degenerate abstract simplicial complexes.

(ii) On the other hand, the set of pairs of non-degenerate abstract simplicial complexes giving rise to isomorphic stable AF-algebras can be effectively enumerated.

(iii) A stable AF-algebra arises from a non-degenerate abstract simplicial complex $\mathcal{C}$ over $\{1,2, \ldots, n\}$ iff $\left(K_{0}(A), K_{0}(A)^{+}\right)$is a finitely generated projective $\ell$ group having infinitely many maximal $\ell$-ideals. This condition is also equivalent to $\left(K_{0}(A), K_{0}(A)^{+}\right)$being a finitely presented $\ell$-group with infinitely many maximal $\ell$-ideals.

Proof. We only have to take care of (iii). By Theorem 3.1 for every non-degenerate abstract simplicial complex $\mathcal{C}$, the dimension group $\lim _{\rightarrow} D(\mathcal{C})$ is isomorphic to $\operatorname{Radiant}(|\Delta(\mathcal{C})|)$. The latter is a finitely generated $\ell$-group with infinitely many maximal $\ell$-ideals.

Claim: Radiant $(|\Delta(\mathcal{C})|)$ is finitely presented.

As a matter of fact, arguing as in the proof of Proposition 2.3 we first extend $\Delta(\mathcal{C})$ to a non-singular fan $\Delta^{\diamond}$ whose support is all of $\mathbf{R}^{n}$, and let $\mathcal{H}\left(\Delta^{\diamond}\right)$ be the set of all Schauder hats of $\Delta^{\diamond}$. We now pick the subset of $\mathcal{H}\left(\Delta^{\diamond}\right)$ given by those hats that are zero over $|\Delta(\mathcal{C})|$. The sum $f$ of the remaining hats is nonzero over $\mathbf{R}^{n} \backslash|\Delta(\mathcal{C})|$ and is constantly zero over $|\Delta(\mathcal{C})|$. Further, by Proposition [2.3(i) (applied to $\Delta^{\diamond}$ ) $f$ is a radiant function over $\mathbf{R}^{n}$, and by Proposition 4.1 there is an $\ell$-group term $\gamma\left(X_{1}, \ldots, X_{n}\right)$ representing $f$ as an element of the free $\ell$-group $F_{n}$, in symbols $f=f_{\gamma}$. The zero set of $f_{\gamma}$ coincides with the support of $\Delta(\mathcal{C})$. The claim is settled.

\footnotetext{
${ }^{12}$ Our present diagrams are the simplification of Bratteli's original definition [3] obtained by stripping all additional machinery needed to code non-stable AF-algebras (such as strong order units, or "scales").
} 
By [1] Theorem 3.1], finitely generated projective $\ell$-groups coincide with finitely presented $\ell$-groups.

Remarks: To the best of our knowledge, there are no "toy" Gödel-incomplete problems.

Every AF-algebra whose Murray-von Neumann order of projections is a lattice, is a quotient of the AF-algebra $M$ described in [13, Section 8]. In particular, every stable AF-algebra whose Bratteli diagram is generated by a non-degenerate abstract simplicial complex is a quotient of $M$.

Let $M_{1}$ be the AF-algebra described in [15, Section 3]. As shown therein, the Effros-Shen AF-algebras $F_{\theta}([\underline{6})$ are precisely the infinite-dimensional simple quotients of $M_{1}$. The tensor product of the AF-algebra $M_{1}$ with the compact operators on a separable Hilbert space has a Bratteli diagram generated by the only non-degenerate abstract simplicial complex over $\{1,2\}$.

The effectiveness of the presentation of the above class of AF-algebras is given by the following result.

Proposition 6.2. For any non-degenerate abstract simplicial complex $\mathcal{C}$, the construction of the diagram $D(\mathcal{C})$ of the stable $A F$-algebra $A(\mathcal{C})$ is effective in the following sense:

(i) There is a Turing machine $\Phi^{\prime}$ which, having as input a non-degenerate abstract simplicial complex $\mathcal{C}$ together with an integer $t \geq 0$, outputs the sequence of $(0,1)$ matrices $\varphi_{0}, \ldots, \varphi_{t}$.

(ii) There is a Turing machine $\Phi^{\prime \prime}$ which, having a non-degenerate abstract simplicial complex $\mathcal{C}$ in its input, together with an arbitrary integer matrix $\varphi$, decides whether $\varphi$ eventually occurs in the sequence (11) - and if this is the case, also outputs the only possible integer $j$ such that $\varphi=\varphi_{j}$.

Proof. (i) The lexicographic order of bra integer vectors, and of pairs of integer vectors, are Turing-decidable relations. The computation of the first bra vector $\mathbf{h}$ having negative temperature for some 2-dimensional cone in $\Delta(\mathcal{C})$ is effective, and so is the determination of (the primitive generating vectors of) the $\mathbf{h}$-coolest cone. Thus the first integer matrix $\varphi_{0}$ can be effectively computed from $\mathcal{C}$. Similarly, there is a Turing machine listing all primitive generating vectors of all cones of the successor $\Delta^{\uplus}(\mathcal{C})$ of the non-singular fan $\Delta(\mathcal{C})$. A direct inspection of the recursive definition of $D(\mathcal{C})$ now yields the desired conclusion for every initial segment of the sequence (1).

(ii) By construction, an integer matrix $M$ will never occur in the sequence (1), unless $M$ is obtainable by a appending a bottom row of the form

$$
0, \ldots, 0,1,0, \ldots, 0,1,0, \ldots, 0 \quad(l \text { entries })
$$

to the $l \times l$ identity matrix. If $M$ does have this form, and $\mathcal{C}$ is an abstract simplicial complex over, say, $\{1, \ldots, n\}$, there is at most one $j$ such that $M$ may satisfy the identity $M=\varphi_{j}$, namely $j=l-n$. Using the Turing machine $\Phi^{\prime}$, we can effectively display the $j$ th initial segment of (1), and then check if the identity holds.

Following [4] and [5], two square, non-singular, integer, primitive ${ }^{13}$ matrices are said to be $C^{*}$-equivalent iff their associated stable AF-algebras are isomorphic.

\footnotetext{
${ }^{13}$ In the sense that sufficiently high powers are strictly positive, i.e., have all entries $>0$.
} 
In [5] it is proved that there is an algorithm to decide isomorphism of stationary AF-algebras arising from primitive matrices.

Proposition 6.3. Consider the following properties, where $f$ ranges over all functions from pairs of abstract simplicial complexes to pairs of square, non-singular, integer, strictly positive matrices:

$P_{1} \quad f$ preserves $C^{*}$-equivalence, i.e., whenever $\mathcal{C}$ and $\mathcal{C}^{\prime}$ are $C^{*}$-equivalent abstract simplicial complexes, then so are the matrices $f(\mathcal{C})$ and $f\left(\mathcal{C}^{\prime}\right)$.

$P_{2} \quad f$ preserves $C^{*}$-inequivalence.

$P_{3} \quad f$ is Turing-computable.

Then for any two distinct indexes $i, j \in\{1,2,3\}$ there is a function $f$ satisfying properties $P_{i}$ and $P_{j}$, but there is no function having simultaneously the three properties.

Proof. Otherwise, if $f$ has all three properties, using the decidability result of [5] we could effectively decide $C^{*}$-equivalence of abstract simplicial complexes, against our main theorem. The rest is clear.

In the introduction of [5] the authors note that their decidability result is interesting in view of the fact that the corresponding problem for non-constant (effectively presented) incidence matrices is undecidable, as shown in [17]. Added weight is given to their remark by Proposition 6.3 because the set of diagrams of the form $D(\mathcal{C})$ is only a simple and small fragment of the class of all effectively presented diagrams.

\section{REFERENCES}

[1] W. M. Beynon, Applications of duality in the theory of finitely generated lattice-ordered abelian groups, Canad. J. Math., 29:243-254, 1977. MR 55:10350

[2] A. Bigard, K. Keimel, and S. Wolfenstein, Groupes et Anneaux Réticulés, Lecture Notes in Mathematics, Vol. 608, Springer-Verlag, New York, 1977. MR 58:27688

[3] O. Bratteli, Inductive limits of finite dimensional $C^{*}$-algebras, Trans. Amer. Math. Soc., 171:195-234, 1972. MR 47:844

[4] O. Bratteli, P. Jorgensen, K. H. Kim, and F. Roush, Non-stationarity of isomorphism between AF-algebras defined by stationary Bratteli diagrams, Ergodic Theory and Dynamical Systems, 20:1639-1656, 2000. MR 2001k:46104

[5] O. Bratteli, P. Jorgensen, K. H. Kim, and F. Roush, Decidability of the isomorphism problem for stationary AF-algebras and the associated ordered simple dimension groups, Ergodic Theory and Dynamical Systems, 21: 1625-1655, 2001. MR 2002h:46088

[6] E. G. Effros, Dimensions and $C^{*}$-algebras, C.B.M.S. Regional Conference Series in Math., Vol. 46, Amer. Math. Soc., Providence, RI, 1981. MR 84k:46042

[7] G. A. Elliott and D. Mundici, A characterisation of lattice-ordered abelian groups, Math. Zeitschrift 213:179-185, 1993. MR 94e:06010

[8] G. Ewald, Combinatorial convexity and algebraic geometry, Graduate Texts in Mathematics, Vol. 168, Springer-Verlag, New York, 1996. MR 97i:52012

[9] A. M. W. Glass and J. J. Madden, The word problem versus the isomorphism problem, J. London Math. Soc., (2), 30:53-61, 1984. MR 86i:03059

[10] A. J. Goldman and A. W. Tucker, Polyhedral convex cones. In Linear equalities and related systems, pp. 19-40, Annals of Mathematics Studies, Vol. 38, Princeton University Press, Princeton, NJ, 1956, MR 19:446g

[11] K. Goodearl, Notes on Real and Complex $C^{*}$-algebras, Birkhäuser, Boston, 1982 (Volume 5 of Shiva Mathematics Series). MR 85d:46079

[12] V. Marra, Every abelian -group is ultrasimplicial, Journal of Algebra, 225:872-884, 2000. MR 2001c:06020 
[13] D. Mundici, Interpretation of $A F C^{*}$-algebras in Eukasiewicz sentential calculus, J. Functional Analysis, 65:15-63, 1986. MR 87k:46146

[14] D. Mundici, Satisfiability in many-valued sentential logic is NP-complete, Theoretical Computer Science, 52:145-153, 1987. MR 89a:68076

[15] D. Mundici, Farey stellar subdivisions, ultrasimplicial groups, and $K_{0}$ of $A F C^{*}$-algebras, Advances in Math., 68:23-39, 1988. MR 89d:46072

[16] D. Mundici, Classes of ultrasimplicial lattice-ordered abelian groups, Journal of Algebra, 213:596-603, 1999. MR 2000d:06023

[17] D. Mundici and G. Panti, The equivalence problem for Bratteli diagrams, Technical Report no. 259, University of Siena (Italy), 1993. Unpublished, 7 pp.

[18] T. Oda, Convex Bodies and Algebraic Geometry, Springer-Verlag, Berlin, 1988. MR 88m:14038

Department of Mathematics "Ulisse Dini", University of Florence, Viale Morgagni 67/A, 50134 Florence, ItAly

E-mail address: mundici@math.unifi.it 